\title{
Modified Asymmetrically-Clipped Optical Orthogonal Frequency-Division Multiplexing System Performance
}

\author{
Salma D. Mohamed, Ivan \\ Andonovic \\ Department of Electronics and Electrical \\ Engineering, \\ Strathclyde University \\ Glasgow, Scotland. \\ salma.mohamed@strath.ac.uk
}

\author{
Hossam Shalaby \\ Department of Electronics and \\ Communications Engineering, \\ Egypt-Japan University of Science and \\ Technology, Alexandria, Egypt, on leave from \\ Electrical Engineering Department, \\ Alexandria University, Alexandria, Egypt.
}

\author{
Moustafa H. Aly \\ College of Engineering, Arab Academy for \\ Science and Technology, Alexandria, \\ Egypt.
}

\begin{abstract}
Improvement in bit error rate performance of asymmetrically-clipped optical orthogonal frequency-division multiplexing (ACO-OFDM) is achieved through proposed modification by using unipolar encoding. This modification eliminates the $3 \mathrm{~dB}$ difference between ACO-OFDM performance and bipolar OFDM.
\end{abstract}

Keywords - asymmetrically-clipped optical orthogonal frequency-division multiplexing (ACO-OFDM); bit error rate (BER).

\section{INTRODUCTION}

In spite of the widespread use of Orthogonal Frequency Division Multiplexing (OFDM) in wireless communications, it has only recently been proposed for use in optical communications [1] principally gated by developments in digital signal processing which have enabled the effective management of optical fiber transmission impairments such as dispersion [1]. Since intensity modulation and direct detection implementations represent the predominant deployments and due to the fact that the OFDM signal is complex, several techniques have been used to convert an OFDM signal into a real, positive domain that then lends itself to direct modulation of the optical source. In order to make the signal truly positive, a number of techniques have been proposed. DC-biased optical OFDM (DCO-OFDM) [2]-[5], asymmetrically-clipped optical OFDM (ACO-OFDM) [2]-[5], and unipolar OFDM (U-OFDM) [5]. In all, Hermitian symmetry is employed such that the output of the IFFT block is real but here a modification to ACO-OFDM is adopted to yield unipolar time encoding instead of zero clipping. Each sample is expressed as a new pair of samples, decreasing the effect of noise on system performance.

\section{SYSTEM MODEL}

The input bits at the transmitter are first mapped to complex M-QAM symbols $A(l)$. These symbols are then input to the IFFT block, the output being complex and bipolar. However as the implementation is founded on intensity modulation, the time domain signal has to be real and positive; the ACOOFDM technique is employed to achieve this [3]-[5]. For an IFFT/FFT of size $N$, the OFDM frame has $N$ subcarriers, so the signal is defined by $A_{\text {frame }}(m), m=0,1,2,3, \ldots, N-1$. In the case of ACO-OFDM, data is only mapped on the odd subcarriers, the even subcarriers being set to zero. Thus only $N / 4$ symbols of $A(l), l=0,1,2,3, \ldots, N / 4-1$ are mapped onto half of the odd subcarriers, $A_{\text {frame }}(m), m=1,3,5, \ldots, N / 2-1$. Hermitian symmetry with $A_{m}=A_{N-m}^{*}$ and $A_{0}=A_{N / 2}=0$ [2]-[5] is then imposed on the other half of the sub-carriers to ensure that the output of the IFFT is real. The ACO-OFDM signal is then passed through the IFFT block, the output time domain signal of the IFFT, $x(n)$ being real but bipolar. This time domain signal is anti-symmetric around the element N/2 (Fig. 1) [4]. In conventional ACO-OFDM, the bipolar real time domain signal $x(n)$ is clipped at the zero level to ensure that the signal is positive; in the proposed system, the unipolar encoding technique suggested by Dobroslav et. al. [5] is used. The unipolar encoding represents each sample of the bipolar time domain signal by a pair of new time samples. If the time sample is positive then the first sample of the new pair is set with its amplitude, and the second sample of the new pair is set to zero. If the original time domain sample is negative, then the first sample of the new pair is set to zero and the second sample of the new pair is set with its positive amplitude (Fig. 2). The optical source is intensity modulated by the modified ACO-OFDM signal $x_{\text {modified } A C O}(n)$ and transmitted through the optical channel subject to AWGN.

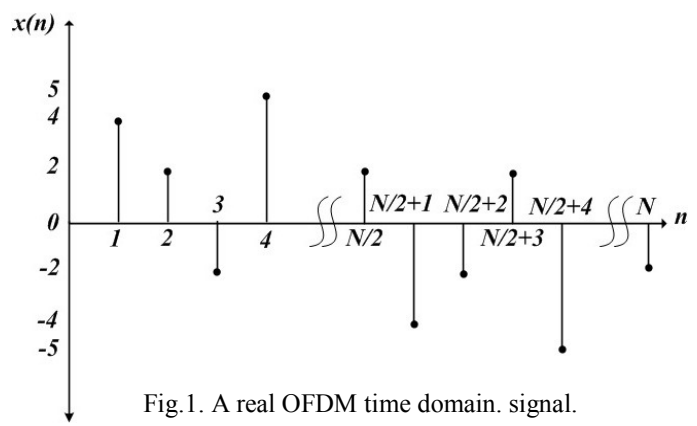

III. SiMULATION RESULTS

Fig. 3 compares the BER performance of the proposed modified ACO-OFDM and conventional ACO-OFDM. 


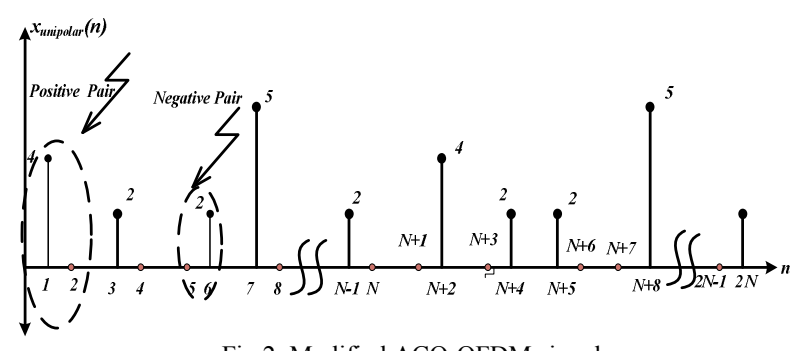

Fig.2. Modified ACO-OFDM signal.

The analysis shows that the performance has improved by nearly $4 \mathrm{~dB}$. Fig. 4 compares the performance to that of bipolar OFDM and that the analysis indicates that the modification has bridged the $3 \mathrm{~dB}$ gap between conventional ACO-OFDM and bipolar OFDM. Fig. 5 shows a comparison between the normalized bandwidth of conventional ACO-OFDM and modified ACO-OFDM for the case of 4, 16, 64, and 256 QAM with respect to the $E_{b(o p t .)} / N_{o}$, where $E_{b(\text { opt. })} / N_{o}=E_{b(\text { elec. })} / \pi N_{o}$, $E_{\text {b(opt.) }}$ is the optical energy per bit, $E_{\text {b(elec.) }}$ is the electrical energy per bit, and $N_{o} / 2$ is the variance of the AWGN.

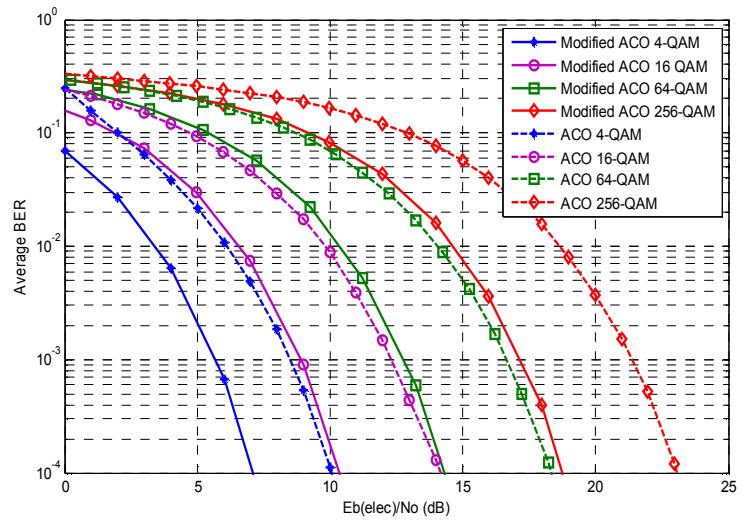

Fig.3 Bit-error rate comparison between the proposed modified ACO- OFDM and conventional ACO-OFDM.

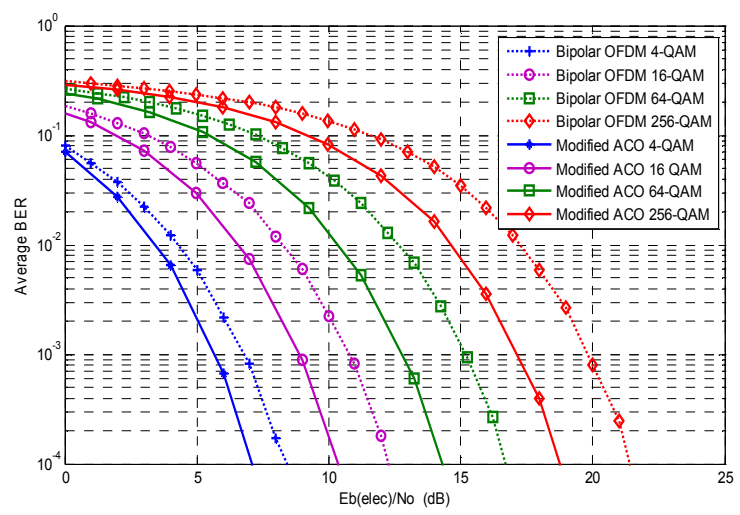

Fig. 4 Bit-error rate comparison between the proposed modified ACO-OFDM and Bipolar OFDM.

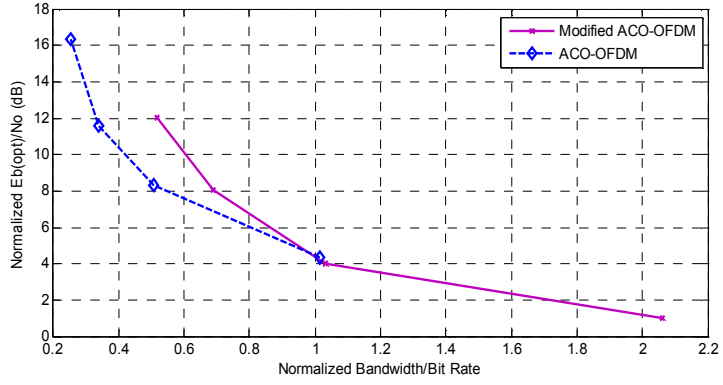

Fig.5 Eb(opt) /No for BER 10-4 and normalized optical power versus normalized bandwidth for ACO-OFDM and modified ACO-OFDM.

The normalized bandwidth in the case of ACO-OFDM is $2(1+2 / N) \log 2 \mathrm{M}$ [4] but as unipolar encoding is used, the length of the OFDM frame is doubled which causes the spectral efficiency to be halved to that of conventional ACOOFDM [5]. Therefore, the normalized bandwidth in the case of modified ACO-OFDM is $4(1+2 / \mathrm{N}) \log 2 \mathrm{M}$. Both 4-QAM ACO-OFDM and 16-QAM modified ACO-OFDM have the same normalized bandwidth and approximately the same optical power. The 4-QAM modified ACO-OFDM requires the least optical power but exhibits the worst bandwidth efficiency.

\section{CONCLUSION}

A modification to conventional ACO-OFDM using unipolar encoding has been proposed. BER performance analysis reveals that the performance of ACO-OFDM can be improved by more than $3 \mathrm{~dB}$ when adopting the proposed approach. These modifications eliminate the reduction in the BER performance between ACO-OFDM and bipolar OFDM.

\section{REFERENCES}

[1] J. Armstrong, "OFDM for Optical Communications," J. Lightwave. Technol., vol. 27, no. 3, pp. 189-204, Feb. 2009.

[2] L. Chen, B. Krongold and J. Evans, "Diversity Combining for Asymmetrically Clipped Optical OFDM in IM/DD Channels," IEEE GLOBECOM, pp. 1-6, Dec. 2009.

[3] S. K. Wilson, and J. Armstrong, "Transmitter and Receiver Methods for Improving Asymmetrically- Clipped Optical OFDM," IEEE Trans. Commun., vol. 8, no. 9, pp. 4561 - 4567, Sep. 2009.

[4] Jean Armstrong and Brendon J. C. Schmidt, "Comparison of Asymmetrically Clipped Optical OFDM and DC-Biased Optical OFDM in AWGN," IEEE Commun. Letters, Vol. 12, no. 5, pp. 343-345, May 2008.

[5] D. Tsonev, S. Sinanovic and H. Haas, "Novel Unipolar Orthogonal Frequency Division Multiplexing (U-OFDM) for Optical Wireless," VTC spring, IEEE $75^{\text {th }}$, pp. 1-5, May 2012. 